\title{
Interplay between habitat fragmentation and climate change: inbreeding affects the response to thermal stress in Drosophila melanogaster
}

\author{
D. Joubert ${ }^{1,2}$, R. Bijlsma ${ }^{1,3, *}$ \\ ${ }^{1}$ Population and Conservation Genetics, ${ }^{2}$ Evolutionary Genetics and ${ }^{3}$ Theoretical Biology, University of Groningen, \\ PO Box 14, 9750 AA Haren, The Netherlands
}

\begin{abstract}
Climate change, exerting thermal stress, and habitat destruction and fragmentation, resulting in genetic drift and inbreeding, are amongst the most disturbing human activities that endanger global biodiversity. We studied the interplay between these 2 processes using Drosophila melanogaster as a model organism and investigated how inbreeding affects the response to thermal stress. We found that both inbreeding and thermal stress negatively affect morphological traits (orbital bristle number, wing width and wing length) as well as a fitness trait (egg-to-adult viability). Most importantly, we observed a significant interaction between inbreeding and thermal stress. Inbred populations suffered considerably more from thermal stress than non-inbred populations for these traits. Moreover, the variation among inbred populations was large: some populations were affected only slightly by thermal stress, but others became extremely sensitive to thermal stress, particularly in egg-to-adult survival. This shows that inbreeding does significantly influence the response to thermal stress, thereby greatly enhancing the effects of temperature stress. We also tested if the effects of inbreeding and thermal stress were reflected in changes in the level of fluctuating asymmetry, in order to determine if this measure can be used as a reliable biomonitor. Although we observed some changes in fluctuating asymmetry under stress, the results are far from unequivocal. We concluded that inbreeding and thermal stress act synergistically and argue that the consequences of climate change and habitat fragmentation should preferably be studied in combination rather than in isolation.
\end{abstract}

KEY WORDS: Thermal stress - Inbreeding - Quantitative variation - Genotype $\times$ environment interaction $\cdot$ Viability $\cdot$ Fluctuating asymmetry $\cdot$ Drosophila melanogaster

\section{INTRODUCTION}

Human activities are rapidly changing both the biotic and abiotic environment at a global scale. Of these activities, climate change, habitat destruction and habitat deterioration are the most conspicuous factors that currently endanger the persistence of biodiversity on earth. The latest IUCN Red List of threatened species (http://www.iucnredlist.org/) shows that out of the 35508 animal species evaluated, 761 have become extinct (documented extinctions since $1500 \mathrm{AD}$ ) or only exist as captive populations and 8782 are threatened in their existence. Furthermore, the combined effects of habitat destruction and environmental stresses such as pollution, global warming and the introduction of exotic species, causing e.g. increased competition or the spread of novel diseases, are expected to increase extinction rates even more in the near future (Reed et al. 2002, Kristensen et al. 2003, Thomas et al. 2004, Hoffmeister et al. 2005, Root \& Schneider 2006).

Climate change has had a significant impact on species and populations in the last 30 to $40 \mathrm{yr}$ (IPCC 2007). Consequences of climate change for natural populations include changes in the distributional range of species, shifts in phenology, changes in community structure and habitat loss (Walther et al. 2002, Mawdsley et 
al. 2009, Chown et al. 2010, this Special). Due to changes in distributional range and loss of habitat, many species (especially endangered species) have been forced to the margin of their distributional range where they often encounter stressful conditions (IPCC 2007, Chown \& Gaston 2008). To survive, individuals in these populations have to be able to adapt to changing and stressful environmental conditions (Hendry et al. 2008). The ability to cope with changing environmental conditions will depend on the amount of genetic variation in the population and the physiological sensitivity of individuals to these environmental changes (Deutsch et al. 2008, Kellermann et al. 2009, Chown et al. 2010). In other words, the ability to adapt to changing conditions will depend on both how well an individual can adjust to the new conditions (Bakker et al. 2010, Canale \& Henry 2010, this Special, de Jong et al. 2010, this Special) and the amount of genetic variation for various fitness traits that is present in the population for evolutionary adaptation to new conditions (Kellermann et al. 2009, Willi \& Hoffmann 2009, Bakker et al. 2010).

Loss of habitat has caused populations of many species to become small and fragmented (Henle et al. 2004, Gaston 2005). In addition to ecological problems, these small, isolated populations will suffer from genetic erosion due to genetic drift and inbreeding. This is often accompanied by inbreeding depression, which causes an increase in extinction risk (Saccheri et al. 1998, Bijlsma et al. 2000, Keller \& Waller 2002, Frankham 2005). The inbreeding and associated inbreeding depression upon population fragmentation may cause population size to decline even more, thereby strengthening the consequences of genetic erosion, resulting in genetic stress. This then will result in the so-called 'extinction vortex' (Gilpin \& Soulé 1986, Brook et al. 2002), ultimately leading to population extinction (Bijlsma et al. 2000). On the other hand, the loss of genetic variation will reduce the ability of small, isolated populations to adapt to changing environmental conditions, i.e. to lower their adaptive potential and thus increase their extinction risk (Frankham 2005, Bell \& Gonzalez 2009, Liao \& Reed 2009, Bakker et al. 2010). Recently it has been shown that especially tropical species and populations will suffer more from climate change in future, as they are highly adapted to their specific environment, which normally shows little fluctuation (Hoffmann et al. 2003, Kellermann et al. 2009).

From a conservation biological perspective, endangered species will be especially vulnerable, as they are prone to both environmental stress and inbreeding depression. In addition, it has been shown that there may be strong synergistic interaction between genetic and environmental stress, leading to greatly elevated extinction probabilities when both stresses coincide (Bijlsma et al. 1999, 2000, 2010, Reed et al. 2002, Kris- tensen et al. 2003, 2008a). The synergistic interaction between genetic and environmental stress has also been observed under semi-natural conditions in the fruit fly Drosophila melanogaster. Kristensen et al. (2008b) showed that inbred flies, compared to noninbred flies, were less likely to locate food patches under warm conditions than under cold conditions. The ability to locate food quickly is important for Drosophila as their food sources are ephemeral and flies can become dehydrated quickly (Kristensen et al. 2008b). Therefore, their study shows that in natural settings inbreeding and thermal stress can also act synergistically, thus increasing the amount of stress on populations and increasing the extinction risk. Good dispersal ability is not only important for rapidly locating food sources, but also to ensure (sufficient) gene flow between fragmented populations. If inbreeding does impede dispersal ability, it will at the same time enhance the effects of fragmentation.

In the present study, we investigated how genetic erosion might affect the response of populations to thermal stress, using Drosophila melanogaster as a model organism. We hypothesized that if inbreeding and temperature stress act synergistically on morphological and fitness traits, the effect of temperature stress on both types of traits should be significantly higher for inbred populations compared to non-inbred populations. To this end, we compared the response of inbred and non-inbred populations to 4 different rearing temperatures: two temperatures that are near the optimal conditions for Drosophila (21 and $25^{\circ} \mathrm{C}$ ) and 2 more extreme or stressful temperatures $\left(17\right.$ and $\left.29^{\circ} \mathrm{C}\right)$. The morphological traits wing length, wing width and orbital bristle number, and the fitness trait egg-to-adult viability were tested, as these traits have previously been shown to be affected by thermal stress (Imasheva et al. 1997, Bijlsma et al. 1999, Bubliy et al. 2000, Joubert 2007). We used a high level of inbreeding ( $F=$ 0.785), which may be higher than generally observed in nature. However, given that the negative effects of inbreeding mostly increase linearly with increasing inbreeding coefficient (Lynch \& Walsh 1998), we argue that comparable results are expected at more ecologically relevant inbreeding levels.

In addition, we investigated a possible change in fluctuating asymmetry (FA) for the morphological characters under both genetic and thermal stress. FA is commonly described as small random deviations from perfect bilateral symmetry in a bilaterally symmetric trait due to 'developmental accidents'. Both genetic (e.g. inbreeding or hybridisation) and environmental stress (e.g. temperature or pollution) are thought to increase the level of FA (Parsons 1992, Clarke 1995). As more individuals may be affected in their development when subjected to increasing levels of stress and, 
consequently, may become more asymmetric, stressed populations are expected to show on average higher levels of FA. Therefore, it has been proposed that the level of stress and FA are positively correlated and that FA may be a good indicator of the presence of stress, possibly being more sensitive than other population survey methods (Clarke 1995, Lens et al. 2002, Hoffmann \& Woods 2003). As such, FA has been put forward as a reliable tool for conservation management (Gilligan et al. 2000, Hoffmann \& Woods 2003, Pertoldi et al. 2006, but see Leung et al. 2003 for critical notes). Here we tested if FA is indeed a measure that signals the presence of stress.

\section{MATERIALS AND METHODS}

\subsection{Lines}

All lines used in the experiments were from the Groningen '83 (G'83) base population that was founded in 1983 with 403 gravid females collected at a fruit market in Groningen, the Netherlands. This population has been maintained since in the laboratory as a large population and has been shown to be genetically highly variable and still contain a considerable genetic load (Bijlsma et al. 1999, 2000). The inbred lines were made by 7 generations of full sib mating leading to a theoretically expected inbreeding coefficient of $F=0.785$. These 10 inbred lines had already been established for a previous experiment (Joubert 2007 ) and maintained as bottle populations ( \pm 300 flies per bottle and 2 bottles per line that were mixed every generation), to keep further inbreeding at a minimum, for 53 generations before the start of this experiment; these are referred to as the inbred populations. On the one hand, this might have allowed for a significant fitness rebound. However, previous experiments have shown this is not likely the case (Bijlsma et al. 2000). On the other hand, new mutations occurring during this period could have decreased the autozygosity levels somewhat (Van Delden \& Beardmore 1968), but we think this should be minimal. Based on the viability difference between non-inbred and inbred populations (see Section 3), we can safely assume that the latter still are highly inbred. As a control (non-inbred), 10 independent non-inbred populations were started by taking subsamples ( \pm 50 pairs) from the base population, 2 generations before the start of the experiments.

\subsection{Rearing conditions}

All lines were maintained in bottles (125 ml volume containing $30 \mathrm{ml}$ standard medium) at standard rear- ing conditions: $25^{\circ} \mathrm{C}, 50 \%$ R.H. and constant light. Standard medium consisted of $1000 \mathrm{ml}$ water, $32 \mathrm{~g}$ dead yeast, $54 \mathrm{~g}$ sucrose, $18 \mathrm{~g}$ agar and $13 \mathrm{ml}$ nipagin solution (10 g nipagin in $100 \mathrm{ml}$ ethanol).

\subsection{Experimental set-up}

To test the effects of temperature stress, genetic stress and the combination of both, the 10 inbred and 10 non-inbred populations were reared at 4 different temperatures: $17,21,25$ and $29^{\circ} \mathrm{C}$. Temperatures of 21 and $25^{\circ} \mathrm{C}$ are near-optimal conditions and 17 and $29^{\circ} \mathrm{C}$ are more extreme temperatures. However, $29^{\circ} \mathrm{C}$ is supposed to be a more stressful temperature than $17^{\circ} \mathrm{C}$ as it is close to the upper temperature boundary for Drosophila melanogaster (e.g. Imasheva et al. 1997, Bijlsma et al. 1999). For each population, 5 bottles were started and incubated at standard rearing conditions. After emerging, the flies were allowed to lay eggs on lids with some standard medium and live yeast for 4 to $5 \mathrm{~h}$. For each population and temperature, 5 bottles, each containing 300 eggs, were established. Bottles for each population were randomly assigned to the different temperatures. The eggs were then incubated at the ascribed temperature. For each population and temperature, flies were collected during peak emergence. Flies from replicate bottles were combined to form one sample. The collected flies were first kept in vials for at least $1 \mathrm{~d}$ to allow their wings to dry properly, and then the females were collected and stored in Eppendorf tubes at $-20^{\circ} \mathrm{C}$ until measuring. The experiment was performed in 2 series, each examining 5 inbred and 5 non-inbred populations. As some of the inbred populations were clearly weak and particularly sensitive to $29^{\circ} \mathrm{C}$ (Bijlsma et al. 1999) and suffered from bacterial infections, a number of populations failed to produce sufficient offspring (at least 30 females) for the FA assay at these conditions. Therefore, these populations were omitted from the experiment, primarily for statistical reasons. Consequently, the results are based on the analyses of 7 inbred and 7 non-inbred populations at each assay condition.

\subsection{Trait measurements}

Of the collected females, a group of 30 individuals per population and temperature were selected for measuring. For each fly, the following traits were either counted or measured on both the left (L) and right (R) sides of the individuals: number of orbital bristles $(\mathrm{OB})$, wing length (WL; length along the third longitudinal wing vein from its intersection with the anterior cross vein to the tip of the wing vein) and wing width (WW; 
length from the tip of the second longitudinal wing vein to the tip of the fifth longitudinal wing vein; as described in Joubert 2007). Both wing traits were measured in $\mu \mathrm{m}$, and all traits were scored and measured by one person (D.J.). Trait size was calculated as ( $\mathrm{L}+$ R)/2. Trait asymmetry (FA) was calculated using the following indices: FA1, which is the mean $|\mathrm{L}-\mathrm{R}|$, and FA4, which is the variance ( $\mathrm{L}-\mathrm{R}$ ) (after Palmer 1994). To get a reliable measure for FA the measurement error should be less that $25 \%$ of the between-sides variance (Palmer 1994). As we knew from previous experiments (Joubert 2007) that the measurement error was generally relatively low, we tested our procedure again here for 4 inbred and 4 non-inbred populations (240 flies) for the 2 wing traits. In all cases, the measurement error was much less than $20 \%$ of the between-sides variance (WL: median $=5.23 \%$, range $=1.43$ to $15.05 \%$; WW: median $=5.48 \%$, range $=2.75$ to $18.58 \%$ ). The measurement error of $\mathrm{OB}$ had in 2 previous experiments (Joubert 2007) already been shown to be low (mean \pm $\mathrm{SE}=7.853 \pm 1.150 \%$ and $4.619 \pm 0.516 \%$, respectively) Therefore, we concluded that our data are not confounded by measurement error and provide a reliable estimate of FA.

\subsection{Viability}

Due to logistic problems, viability of the inbred and non-inbred populations could not be determined during the experiment. Therefore, 2 mo later the viability of the 7 inbred populations and, unfortunately, of only 3 of the original non-inbred populations were assayed for all 4 temperatures. Five bottles per population and temperature, each containing 300 eggs (as described for the experimental set-up), were established. The flies were collected at regular time intervals until all flies had emerged, and the number of flies that emerged was counted and used as the measure of viability (no. adults/no. eggs).

\subsection{Statistical analysis}

The FA values of the different traits were first tested per population and temperature for departures from normality, directional asymmetry and size dependence, which are known to inflate FA. The results were then tested as proposed by Palmer (1994). To all analyses done within a set (e.g. all tests for departures from normality within a population and temperature), sequential Bonferroni correction was applied (Rice 1989).

To test for the effects of inbreeding level and temperature on the morphological traits, we used nested ANOVAs with populations nested within breeding regime and temperature as a random effect. Viabilities were analyzed using untransformed data as the results for arcsine square root-transformed data were qualitatively the same (data not shown).

\section{RESULTS}

\subsection{Quantitative characters: trait size and variation}

Not surprisingly, the reaction norms showed that trait size varied significantly with breeding temperature (Fig. 1, Table 1). WL and WW decreased continuously with increasing temperature for both inbred and noninbred populations (Fig. 1C-F). Both traits are known to be strongly correlated with body size which, in turn, is well known to decrease significantly with increasing developmental temperatures (e.g. Karan et al. 1998, Gibert et al. 2004). OB number, on the other hand, showed an optimum at $21^{\circ} \mathrm{C}$ with lower numbers at the other temperatures (Fig. 1A, B). This is not unlike what is generally found for bristle characters (e.g. Moreteau et al. 2003, Gibert et al. 2004). Both WL and WW mean trait sizes were significantly affected by inbreeding (Table 1). In contrast, OB did not show a significant inbreeding effect. However, the substantial betweenpopulation variation in mean trait size observed for this bristle trait both among inbred and non-inbred populations (Fig. 1) may have decreased the power of our statistical test. Results of the nested ANOVA showed that trait size indeed differed significantly between individual populations for $\mathrm{OB}$, and that populations also differed significantly for the 2 wing traits (Table 1). In addition, the variation among populations was larger among the inbred populations than among the noninbred populations for all 3 traits (Fig. 1). This was confirmed by the results of a 2-way ANOVA on the data for the non-inbred and inbred populations separately, which showed that the variation in mean trait size for all traits was mainly due to the much greater variation among the inbred populations compared to the noninbred populations (data not shown). The higher variance among inbred populations is partly explained by the repeated founder events the inbred populations have undergone during the inbreeding procedure.

Much more important, however, are the significant interactions between both inbreeding and temperature and between population and temperature (Table 1). This shows not only that inbred populations, in general, are more sensitive to changes in temperature but also that different inbred populations react differently to these changes. The panels of Fig. 1 clearly illustrate this difference in the reaction of inbred populations as the reaction norms of the individual inbred populations cross. These results show that changes in thermal envi- 

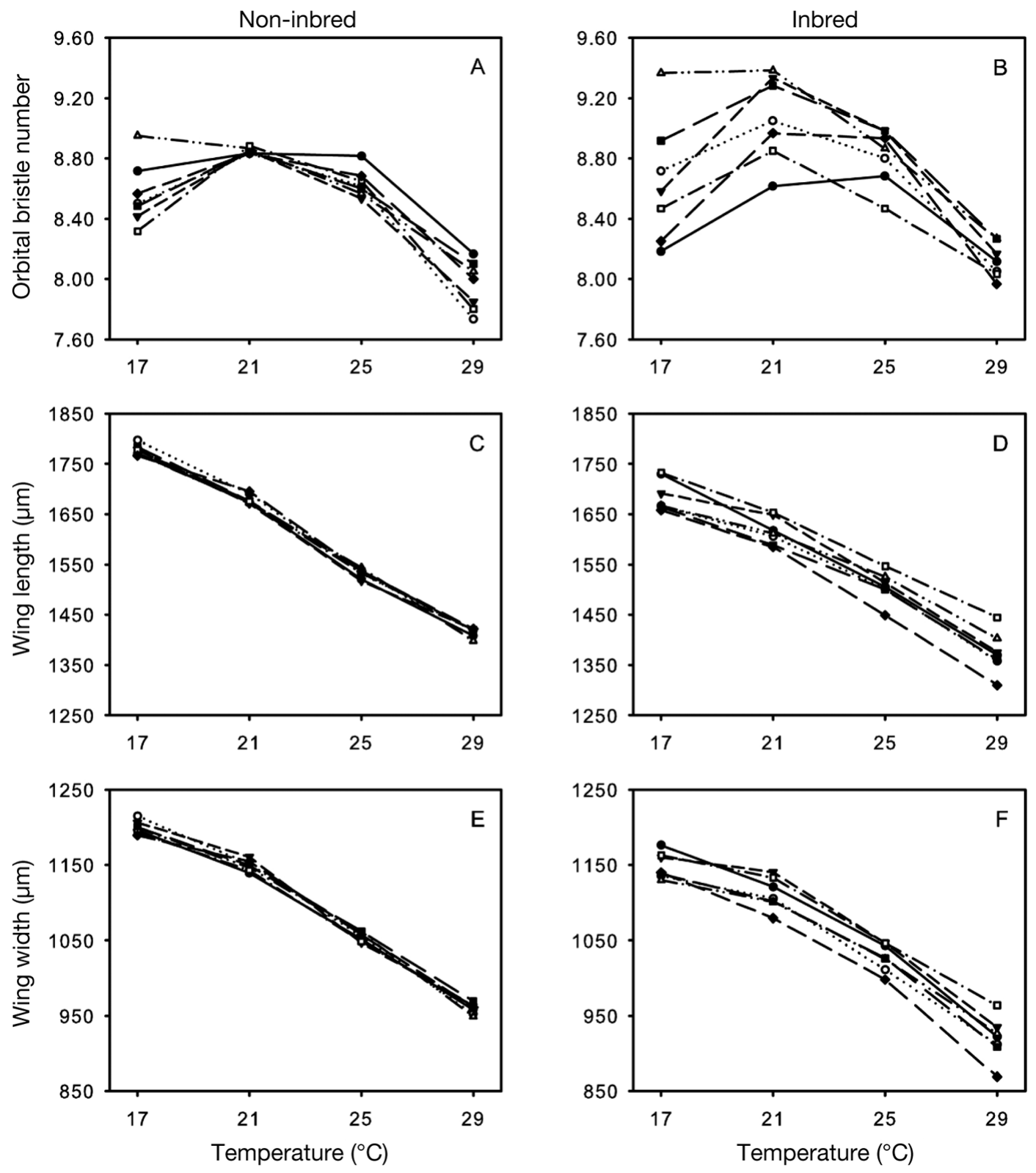

Fig. 1. Drosophila melanogaster. Mean trait values given per population and temperature. (AC,E) non-inbred populations; $(\mathrm{B}, \mathrm{D}, \mathrm{F})$ inbred populations. $(\mathrm{A}, \mathrm{B})$ Mean orbital bristle number; $(\mathrm{C}, \mathrm{D})$ mean wing length; $(\mathrm{E}, \mathrm{F})$ mean wing width. Different symbols/lines indicate individual populations

ronment and environmental perturbations will have significantly different consequences for inbred populations than for non-inbred populations.

If inbred populations are more sensitive to thermal stress they may have difficulties developing consistently in a stressful environment, and this interaction between inbreeding and environment could increase the phenotypic variance within populations (Woods et al. 1999, Fowler \& Whitlock 2002). The coefficient of variation $(\mathrm{CV})$ of a trait is commonly used to assess the amount of variation within a population. $\mathrm{CV}$ values for OB number were clearly much higher in both the non- inbred and inbred populations compared to the $\mathrm{CV}$ values of the other 2 traits (Fig. 2). In many cases, OB $\mathrm{CV}$ showed the lowest values at 21 to $25^{\circ} \mathrm{C}$ and higher levels at 17 and $29^{\circ} \mathrm{C}$ (Fig. 2). Furthermore, Fig. 2 shows that there is substantial variation between populations in $\mathrm{OB} \mathrm{CV}$, especially at 17 and $29^{\circ} \mathrm{C}$. However, neither the 42 tests comparing the mean $\mathrm{CV}$ values between temperatures within populations (data not shown) nor the tests for homogeneity $\left(\chi^{2}\right.$-test for homogeneity; Zar 1996) of CV values among separate populations within a temperature (Table 2) were significant for this trait. For WL and WW, inbred populations 
Table 1. Nested ANOVAs (Drosophila melanogaster populations nested within inbreeding level) testing for differences in mean trait size between temperatures, inbreeding levels and their interactions. OB: orbital bristle number; WL: wing length; WW wing width

\begin{tabular}{lcrrr}
$\begin{array}{l}\text { Trait } \\
\text { Source of variation }\end{array}$ & df & \multicolumn{1}{c}{ MS } & $F$ & \multicolumn{1}{c}{$\mathrm{p}$} \\
\hline OB & & & & \\
Inbreeding level (A) & 1 & 10.768 & 3.287 & 0.095 \\
Temperature (B) & 3 & 64.025 & 81.198 & $<0.001$ \\
Population (C) & 12 & 3.276 & 9.110 & $<0.001$ \\
A $\times$ B & 3 & 0.381 & 0.484 & 0.696 \\
B $\times$ C & 36 & 0.789 & 2.192 & $<0.001$ \\
Error & 1624 & 0.360 & & \\
& & & & \\
Source of variation & $\mathrm{df}$ & $\mathrm{MS} \times 10^{3}$ & $F$ & $\mathrm{p}$ \\
\hline WL & & & & \\
Inbreeding level (A) & 1 & 1281.183 & 23.859 & $<0.001$ \\
Temperature (B) & 3 & 9202.550 & 1569.552 & $<0.001$ \\
Population (C) & 12 & 53.697 & 37.655 & $<0.001$ \\
A $\times$ B & 3 & 84.391 & 14.393 & $<0.001$ \\
B $\times$ C & 36 & 5.863 & 4.112 & $<0.001$ \\
Error & 1624 & 1.426 & & \\
WW & & & & \\
Inbreeding level (A) & 1 & 628.778 & 26.647 & $<0.001$ \\
Temperature (B) & 3 & 4537.545 & 1633.101 & $<0.001$ \\
Population (C) & 12 & 23.597 & 32.586 & $<0.001$ \\
A $\times$ B & 3 & 10.542 & 3.794 & 0.018 \\
$B \times$ C & 36 & 2.778 & 3.837 & $<0.001$ \\
Error & 1624 & 0.724 & & \\
\hline
\end{tabular}

clearly showed much more variation in CVs compared to the non-inbred populations (Fig. 2). A test for homogeneity of $\mathrm{CV}$ values among populations within a temperature confirmed this higher variation in the inbred populations for all temperatures tested (Table 2). Interestingly, for both wing traits, one inbred population gave an extreme value at $17^{\circ} \mathrm{C}$ (Fig. 2), indicating that for that particular population, $17^{\circ} \mathrm{C}$ may represent extreme conditions, inflating the variation among individuals. Non-inbred populations, however, showed a significant among populations variation in CV for WL at $17^{\circ} \mathrm{C}$ only, while all other tests for homogeneity among non-inbred populations were non-significant (Table 2). In conclusion, the CV results show that inbred populations are significantly more sensitive to thermal stress compared to non-inbred populations.

\subsection{Viability and temperature}

Fitness (related) traits (e.g. viability, extinction risk) have previously been shown to be affected by both inbreeding and thermal stress (Bijlsma et al. 1999, 2000, Dahlgaard \& Hoffmann 2000, Armbruster \& Reed 2005, Pedersen et al. 2005, Joubert 2007). In Fig. 3A, the viability of the 3 non-inbred and 7 inbred populations is shown. As expected, inbred populations clearly have a lower viability compared to non-inbred populations. All inbred populations showed lower viability compared to the non-inbred populations at all temperatures, while some inbred populations showed very low viability (Fig. 3A). Averaged over all lines, the inbred populations showed a reduction in viability of $64 \%$ (average viability $\pm \mathrm{SE}=0.238 \pm 0.032$ and $0.667 \pm 0.027$, for inbred and non-inbred populations, respectively). A $t$-test comparing inbred populations with non-inbred populations (equal variances not assumed) shows that inbred populations have a significantly lower viability compared to non-inbred populations at all temperatures $\left(t\right.$-test, $17^{\circ} \mathrm{C}: t=4.675, \mathrm{df}=$ 6.923, $\mathrm{p}=0.002 ; 21^{\circ} \mathrm{C}: t=4.763, \mathrm{df}=7.501, \mathrm{p}=0.002$; $25^{\circ} \mathrm{C}: t=5.551, \mathrm{df}=7.559, \mathrm{p}=0.001 ; 29^{\circ} \mathrm{C}: t=4.940$, $\mathrm{df}=6.297, \mathrm{p}=0.002)$. The variation among populations appears to be larger for inbred populations than for non-inbred populations. However, a test for homogeneity showed that this difference was not significant, most likely due to the unbalanced design (data not shown). More importantly, temperature had a significant effect on the viability of both the non-inbred and inbred populations (ANOVA, non-inbred populations: $F_{3,6}=10.372, \mathrm{p}=0.009$; inbred populations: $F_{3,18}=$ 15.661, $\mathrm{p}=0.001)$. In this case, the viability was the lowest for flies reared under the extreme temperatures $\left(17\right.$ and $29^{\circ} \mathrm{C}$ ) and highest for flies reared under the more optimal conditions ( 21 and $25^{\circ} \mathrm{C}_{i}$ Fig. $3 \mathrm{~A}$ ).

The difference in mean viability among populations shown in Fig. 3A may obscure the changes in viability in relation to temperature, especially when viability is low as observed for some inbred populations. Therefore, for each population we calculated the viability of each temperature relative to the highest average viability observed for that population (adjusted viability). As such, Fig. 3B shows the effect of temperature relative to the temperature at which the highest viability was observed. The highest viability was generally observed for the intermediate temperatures $\left(21\right.$ and $\left.25^{\circ} \mathrm{C}\right)$, while viability decreased towards the extremes. Importantly, this decrease was substantially larger for the inbred populations than for the non-inbred populations (Mann Whitney $U$-test, non-inbred versus inbred, $17^{\circ} \mathrm{C}: Z=$ $-1.709, \mathrm{p}=0.087 ; 21^{\circ} \mathrm{C}: Z=-0.122, \mathrm{p}=0.903 ; 25^{\circ} \mathrm{C}: Z=$ $-0.851, \mathrm{p}=0.395 ; 29^{\circ} \mathrm{C}: Z=-2.393, \mathrm{p}=0.017$ ). Two of the inbred populations showed a very strong decrease when tested at 29 and $17^{\circ} \mathrm{C}$. This shows that inbreeding not only compromises viability (fitness) but that this effect becomes enhanced under stress conditions.

\subsection{Effects of inbreeding and temperature stress on FA}

It has been proposed that the level of stress and FA are positively correlated and that FA may be a good 

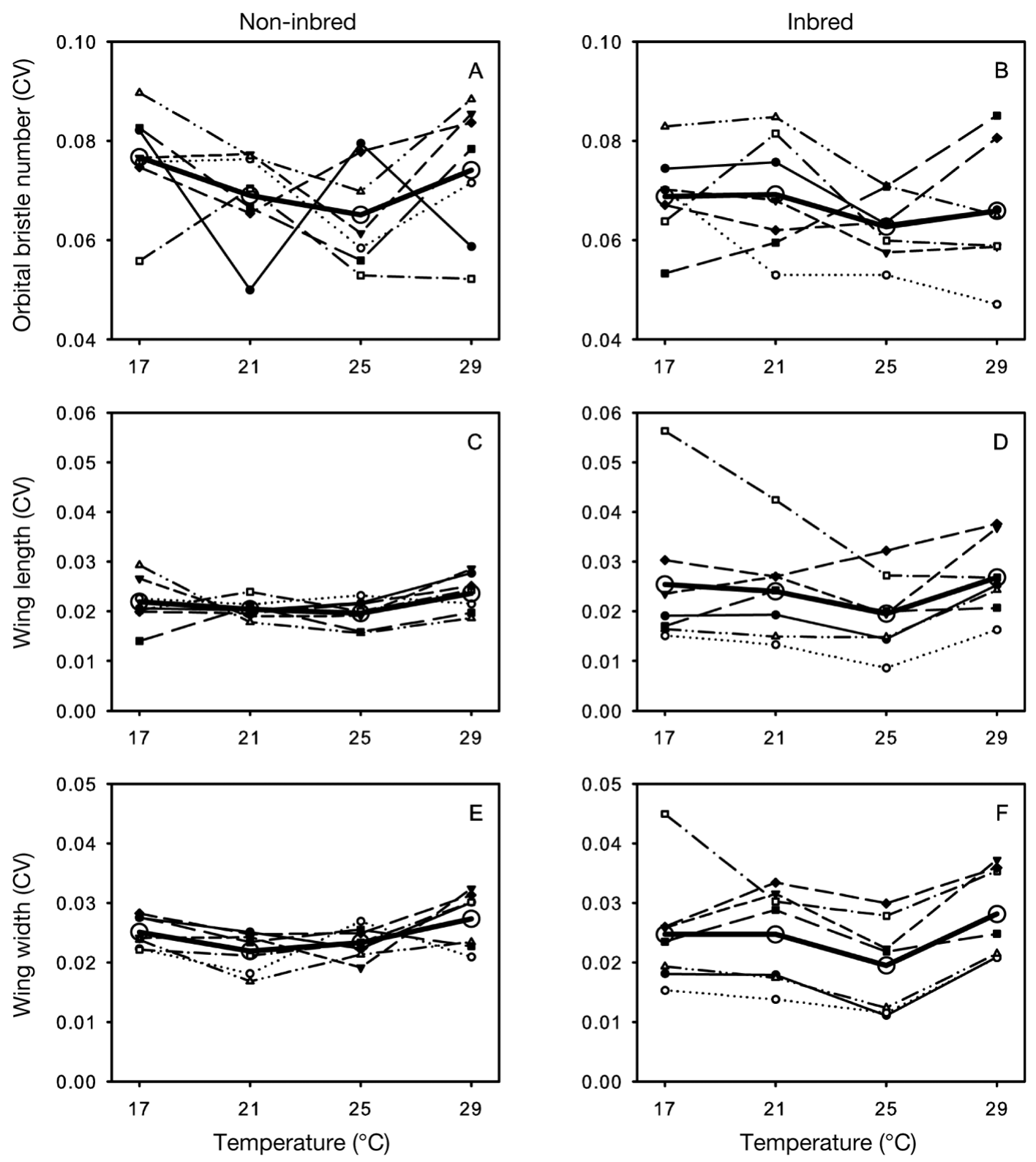

Fig. 2. Trait size coefficients of variation (CVs) given per Drosophila melanogaster population and temperature. The open circles and thick line show the grand mean of the 7 populations. $(A, C, E)$ non-inbred populations; $(B, D, F)$ inbred populations. $(A, B)$ Orbital bristle number $\mathrm{CV}_{i}(\mathrm{C}, \mathrm{D})$ wing length $\mathrm{CV}_{i}(\mathrm{E}, \mathrm{F})$ wing width $\mathrm{CV}$. Different symbols/lines indicate individual populations

indicator of the presence of stress. We therefore tested the effects of both inbreeding and temperature stress on levels of trait asymmetry for the 3 bilateral traits (OB, WL and WW), to assess if FA can be used as an indicator of the presence of either environmental or genetic stress.

Departures from normality, the presence of directional asymmetry and size dependence of asymmetry all can significantly inflate FA values. Consequently, we first tested our data for these factors before analysing the asymmetry data and found that we did not have to correct our data for these factors (data not shown). Additionally, we calculated 2 different asymmetry indices (FA1 and FA4) to test for the effects of inbreeding and temperature stress. As the results of both indices were comparable, we here report the results of FA1 only.

Differences in FA1 between temperatures and inbreeding levels were tested by means of a nested ANOVA (populations nested within inbreeding level). A significant effect of inbreeding was observed for WL FA1 (nested ANOVA, inbreeding versus population: 
Table 2. Results of the test for homogeneity of Drosophila melanogaster population coefficients of variation within a temperature (Zar 1996). $\chi^{2}$ values and significance levels, per trait, temperature and breeding group, are given. OB: orbital bristle number; WL: wing length; WW: wing width. Numbers in bold indicate tests that were significant after Bonferroni correction (corrected for the 4 tests within each breeding $\times$ trait group)

\begin{tabular}{|lccccc|}
\hline \multirow{2}{*}{$\begin{array}{l}\text { Trait } \\
\text { Temp. }\left({ }^{\circ} \mathrm{C}\right)\end{array}$} & $\mathrm{df}$ & $\chi^{2}$ & $\mathrm{p}$ & $\chi^{2}$ & $\mathrm{p}$ \\
\hline OB & & & & & \\
17 & 6 & 6.569 & 0.363 & 6.086 & 0.414 \\
21 & 6 & 6.811 & 0.339 & 10.132 & 0.119 \\
25 & 6 & 9.262 & 0.159 & 3.864 & 0.659 \\
29 & 6 & 12.240 & 0.057 & 13.797 & 0.032 \\
WL & & & & & \\
17 & 6 & $\mathbf{1 7 . 9 2 9}$ & $\mathbf{0 . 0 0 6}$ & $\mathbf{1 1 5 . 1 7 9}$ & $<\mathbf{0 . 0 0 1}$ \\
21 & 6 & 3.164 & 0.788 & $\mathbf{5 8 . 0 3 8}$ & $<\mathbf{0 . 0 0 1}$ \\
25 & 6 & 7.683 & 0.262 & $\mathbf{5 8 . 7 2 3}$ & $<\mathbf{0 . 0 0 1}$ \\
29 & 6 & 9.237 & 0.161 & $\mathbf{3 0 . 0 5 6}$ & $<\mathbf{0 . 0 0 1}$ \\
$\mathbf{W W}$ & & & & & \\
17 & 6 & 3.825 & 0.700 & $\mathbf{5 4 . 3 9 7}$ & $<\mathbf{0 . 0 0 1}$ \\
21 & 6 & 8.032 & 0.236 & $\mathbf{3 6 . 8 1 9}$ & $<\mathbf{0 . 0 0 1}$ \\
25 & 6 & 4.533 & 0.605 & $\mathbf{5 6 . 9 8 2}$ & $<\mathbf{0 . 0 0 1}$ \\
29 & 6 & 10.420 & 0.108 & $\mathbf{2 6 . 0 2 4}$ & $<\mathbf{0 . 0 0 1}$ \\
\hline
\end{tabular}

$\left.F_{1,12}=13.792, \mathrm{p}=0.003\right)$, but not for the other 2 traits (OB: $F_{1,12}=0.730, \mathrm{p}>0.05$; WW: $F_{1,12}=2.420, \mathrm{p}>0.05$ ). This is also clearly seen in Fig. 4B, which shows that the inbred populations were more asymmetric compared to the non-inbred populations at all temperatures, while Figs. 4A and $\mathrm{C}$ show little to no consistent differences between inbred and non-inbred populations. For $\mathrm{OB}$ and WW, however, the figures suggest that the difference in FA is the largest at $29^{\circ} \mathrm{C}$. On the other hand, temperature only significantly affected WW (nested ANOVA, temperature versus temperature-population interaction: $F_{3,36}=6.870, \mathrm{p}=0.001$ ), in which case WW FA1 was minimal at $25^{\circ} \mathrm{C}$ and significantly increased towards the extreme temperatures (Fig. 4C), again suggesting that the extreme temperatures are indeed stressful. OB and WL asymmetry both showed significant between-population differences (nested ANOVA, population versus error component, OB: $F_{12,1624}=2.125, \mathrm{p}=0.013$; WL: $F_{12,1624}=2.061, \mathrm{p}=$ $0.017)$. On the other hand, WW did not show such an effect. These differences in population effects between traits indicate that trait asymmetry was not always affected by between population differences as compared to mean trait size, which did show significant among-population differences for all traits. None of the 3 traits showed a significant interaction between temperature and inbreeding (data not shown). As a significant population effect was observed for both $\mathrm{OB}$ and WL asymmetry, we conducted a 2-way ANOVA (temperature by population) on the inbred and non-inbred
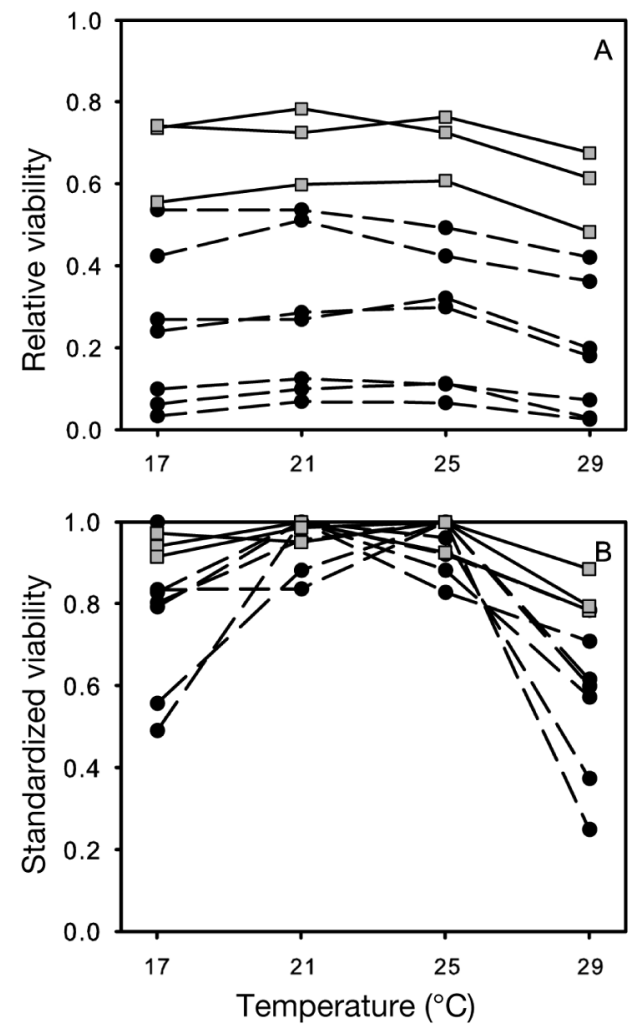

Fig. 3. Drosophila melanogaster. Viability (egg-to-adult viability) given per population and temperature for non-inbred (grey squares) and inbred populations (black circles). (A) Mean or relative viability (calculated as the average over 5 vials per population and temperature). (B) Standardized viability (calculated as the viability of each temperature relative to the highest average viability observed for that population)

groups separately to test for a possible difference in response within the 2 groups. The results showed that the significant difference between populations for OB and WL was due to differences between inbred populations, as in both cases the variation among inbred populations was higher than for non-inbred populations (2-way ANOVA, population versus error component, OB: inbred, $F_{6,812}=2.280, \mathrm{p}=0.034$; noninbred, $F_{6,812}=1.940, \mathrm{p}>0.05 ;$ WL: inbred, $F_{6,812}=$ $2.788, \mathrm{p}=0.011$; non-inbred, $\left.F_{6,812}=0.940, \mathrm{p}>0.05\right)$. As suggested by Fig. 4B, a significant temperature effect was found for WL FA1 in the non-inbred populations only (2-way ANOVA, temperature versus interaction term, $F_{3,18}=4.469, \mathrm{p}=0.016$ compared to $F_{3,18}=$ $0.155, \mathrm{p}>0.05$ for inbred populations). In this case, WL asymmetry was highest at $29^{\circ} \mathrm{C}$ and was significantly different compared to the FA1 value at $21^{\circ} \mathrm{C}$ (Tukey HSD, mean difference $=2.845, \mathrm{p}=0.012$ ). The relatively high variation among inbred populations (as indicated by the significant population term in the 2-way ANOVA) could have obscured a significant temperature effect in the total ANOVA. The significant 

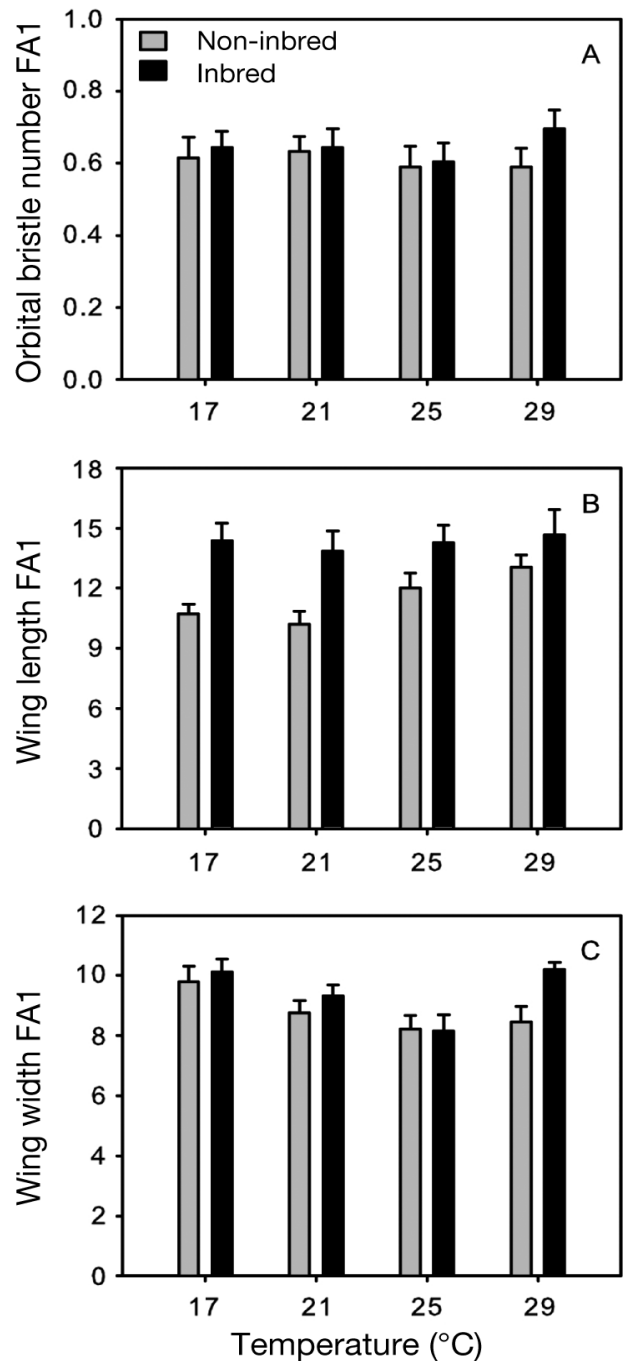

Fig. 4. Mean \pm SE fluctuating asymmetry (FA1) values per temperature. Mean FA1 values were calculated from the population means of either the non-inbred or the inbred Drosophila melanogaster populations for (A) orbital bristle number, (B) wing length and (C) wing width

effect of temperature on WW FA1 was mainly caused by a significant temperature effect for the inbred populations (2-way ANOVA, temperature against interaction term, $F_{3,18}=6.186, \mathrm{p}=0.004$ compared to $F_{3,18}=$ 2.858, p > 0.05 for non-inbred populations). At 29 and $17^{\circ} \mathrm{C}$, WW FA1 was significantly higher compared to WW FA1 at $25^{\circ} \mathrm{C}$ (Tukey HSD, mean difference = 2.057, $\mathrm{p}=0.034$ and mean difference $=1.977, \mathrm{p}=0.045$, respectively). Particularly, inbred populations were more sensitive to more extreme temperatures, as was found for mean trait size (Table 1). In conclusion, the results show that both inbreeding and temperature stress had an effect on trait FA. As we did not observe a significant interaction between temperature and inbreeding, no obvious synergism seems to occur between both stresses for FA: inbreeding only affected
WL asymmetry, while temperature only affected WW FA. Importantly, however, the FA results did clearly indicate that inbred populations were more sensitive to the extreme temperatures.

\section{DISCUSSION}

\subsection{Quantitative traits: inbreeding and temperature effects}

The effect of inbreeding on quantitative traits can be quite complex, as inbreeding may affect the average expression of traits, the amount of genetic and environmental variance of traits and the variance among lines (Whitlock \& Fowler 1999, Fowler \& Whitlock 2002, Van Buskirk \& Willi 2006). Similar to other studies (Whitlock \& Fowler 1999, Fowler \& Whitlock 2002), we observed that average trait size decreased, at least for WW and WL, showing that our inbreeding procedures did affect quantitative variation. Moreover, the variation in average trait size among populations was found to be significantly higher for the inbred populations compared to the non-inbred populations (Fig. 1). This is to be expected, as inbreeding is accompanied by genetic drift that causes fixation of different alleles in different populations. As such, the variation within the original base population will become redistributed as among-population variation. This has been observed in many previous studies of Drosophila melanogaster (Whitlock \& Fowler 1999, Fernández et al. 2003, Van Buskirk \& Willi 2006).

Inbreeding is also expected to affect the phenotypic variation within lines. Because of the inbreeding process, the additive genetic variation within populations will become depleted and, in the absence of dominance, the genetic variance $\left(\mathrm{V}_{\mathrm{G}}\right)$ will decrease significantly (Falconer \& Mackay 1996). Consequently, the total phenotypic variance $\left(\mathrm{V}_{\mathrm{p}} ; \mathrm{V}_{\mathrm{p}}=\mathrm{V}_{\mathrm{G}}+\mathrm{V}_{\mathrm{E}} \mathrm{V}_{\mathrm{E}}\right.$ being the environmental variance) is also expected to decrease. Assuming the variance for these quantitative traits acts mostly additively, we would expect a decrease in the phenotypic variance within inbred populations. However, we observed considerable variation, particularly among inbred populations, for the withinpopulation CVs (which were often highly inflated), especially for the 2 wing traits (Table 2, Fig. 2). Comparable results were observed by Fowler \& Whitlock (1999) and Whitlock \& Fowler (1999), who also found significant increases in phenotypic variance within inbred lines for several wing traits. One has to realize, however, that the level of phenotypic variation within inbred populations depends on the balance between the decrease in additive genetic variance, as discussed above, and an increase in the environmental variance 
$\left(\mathrm{V}_{\mathrm{E}}\right)$. It is well documented that for many different organisms (e.g. Drosophila, mice, rats, chicken and maize) the phenotypic variance observed for inbred lines is generally much higher than for F1s from crosses between different highly inbred lines. This has been attributed to a difference in the environmental variance component $\left(\mathrm{V}_{\mathrm{E}}\right)$, as both the inbred lines and their crosses have near zero genetic variances. Many examples of this finding can be found in Lerner (1954), Falconer \& Mackay (1996) and Wright (1977). The cause of the elevated environmental variance in inbred populations is still not well understood, but it seems clear that inbred populations show decreased developmental homeostasis (Lerner 1954, Waddington 1957, Falconer \& Mackay 1996). This fits well with recent findings that inbred populations are generally much more susceptible to environmental perturbations (Bijlsma et al. 1999, 2000, Reed et al. 2002, Kristensen et al. 2003, Pedersen et al. 2005).

The temperature that an organism encounters during its development has a large effect on the development of its traits, as the developmental time greatly increases when temperatures decrease. As body size is positively correlated with developmental time (Zwaan et al. 1995, Nunney 1996), we expected the size characters WL and WW to be affected by this relationship and to show a strong negative correlation with breeding temperature. Fig. 1 shows that this is clearly the case. The reaction norms for the 2 wing size measures and for OB numbers of both the inbred and non-inbred populations show similar shapes, as found in other studies on Drosophila melanogaster using the same or comparable traits (e.g. Imasheva et al. 1998, Gibert et al. 2004, Kjærsgaard et al. 2010, this Special, Trotta et al. 2010, this Special).

Apart from the effects on mean trait size, temperature also affected the variation among populations. Figs. 1 (mean trait size) and 2 (trait CV) clearly show that the reaction norms for inbred populations are more variable compared to those for non-inbred populations. Furthermore, we observed a significant population-temperature interaction for all 3 traits (Table 1), which was especially apparent for the inbred populations (Figs. $1 \& 2$ ). The large differences in form of the reaction norms for inbred populations (particularly for WW and WL) show that inbred populations in particular differ greatly in their sensitivity to temperature change. Interestingly, one of the inbred populations showed a conspicuous temperature sensitivity when reared at $17^{\circ} \mathrm{C}$ as the within-line $\mathrm{CV}$ for both wing traits increased greatly at this temperature (Fig. 2D, F). Such a striking genotype $\times$ environment interaction has been observed before for inbred lines obtained from the same base population. For instance, Vermeulen \& Bijlsma (2004a,b) showed that lifespan became greatly curtailed at extreme temperatures in specific inbred lines caused by temperature-sensitive alleles. A comparable effect has recently been observed for pupal survival (Bijlsma et al. 2010)

The results also showed a tendency for an increase in variation in trait size among individuals within populations at the extreme temperatures (Fig. 2). This tendency is clearer for inbred populations, which showed higher and more variable CV values at the extreme temperatures 17 and $29^{\circ} \mathrm{C}$ (Fig. 2, Table 2). A higher $\mathrm{CV}$ at extreme temperatures for inbred lines is what one would expect, as trait CVs are said to increase in a stressful environment, especially for inbred individuals (e.g. Woods et al. 1999). As discussed above, this can be attributed to the fact that inbred populations are more sensitive to environmental perturbations (Bijlsma et al. 1999, Reed et al. 2002, Kristensen et al. 2003, 2008a). These results show that the populations experienced both thermal and genetic stress.

As such, we observed a significant interaction between genetic erosion and thermal stress as inbred populations in particular were more sensitive to changes in temperature and showed greater variation in reaction norms. This interaction is of great importance for natural populations, as our results show that changes in the thermal environment are experienced differently by different inbred populations compared to non-inbred populations. In addition, inbred populations were found to be much more sensitive to temperature change, particularly when experiencing extreme temperatures.

\subsection{Viability under thermal and genetic stress}

The discussion in the previous section focussed on traits that are only indirectly related to fitness. We also measured viability to assess the effects of genetic erosion and thermal stress on a fitness trait. Not surprisingly, inbreeding was found to have a much more extreme effect on viability. Although some inbred populations show a viability nearly similar to the noninbred populations, most have a greatly decreased fitness. It is generally observed that traits directly related to fitness exhibit much higher levels of inbreeding depression than morphological traits, as the genetic variance of the former is mainly governed by dominance, while that of the latter is mainly additive (Mousseau \& Roff 1987, Lynch \& Walsh 1998).

Temperature also had a significant effect on viability, as the fitness was lower at extreme temperatures, particularly at $29^{\circ} \mathrm{C}$ (Fig. 3), indicating that these temperatures $\left(17\right.$ and $\left.29^{\circ} \mathrm{C}\right)$ exert thermal stress. Other studies have shown more or less the same relation, and have indicated that the optimal temperature for 
Drosophila melanogaster is around 22 to $23^{\circ} \mathrm{C}$ (David et al. 1983, R. Bijlsma unpubl. data). However, when viability was standardized, we observed a much stronger decrease in viability at the extreme temperatures for inbred populations compared to non-inbred populations (Fig. 4). Some inbred populations showed a more than $50 \%$ reduction in viability when cultured at extreme temperatures. As for morphological characters, inbred populations seemed to suffer much more from thermal stress also with respect to viability. A higher sensitivity to thermal stress of inbred populations has now been found in several other studies and is associated with an increase in the extinction risk of inbred populations (Bijlsma et al. 2000, Reed et al. 2002, Kristensen et al. 2003, 2008c, Pedersen et al. 2005). This may imply that small, isolated populations will have increased difficulty in coping successfully with the more extreme temperatures caused by climate change, ultimately causing these populations to become extinct.

\subsection{FA under inbreeding and thermal stress}

It would be practical if the presence of either thermal or genetic stress, or both, would be reflected by an increase in the level of FA. As such, changes in FA could possibly serve to signal the presence of such stresses under natural conditions.

Both the quantitative traits and viability show that inbred individuals are less able to maintain their optimal phenotype under stress conditions; in other words, they are less able to buffer their development under stress. As FA is thought to reflect developmental stability (Palmer 1994, Clarke 1995), we might expect FA to increase as a result of inbreeding and thermal stress. Given our observations that inbred populations in particular suffered from thermal stress, we would expect increased FA when genetic and thermal stresses coincide. Both stresses were observed to increase FA, but each stress increased a different trait. Inbreeding affected WL asymmetry, while thermal stress had an effect on WW asymmetry only (Fig. 4). The trait specificity of stress-asymmetry associations as observed here has also been observed in other studies (Vøllestad et al. 1999, Woods et al. 1999, Hoffmann \& Woods 2003).

More importantly and contrary to expectations, no interaction between inbreeding and thermal stress was observed for any of the 3 morphological characters studied. This agrees well with the results of several other studies that have tested for the possible relation between the combined stresses and changes in FA (Hosken et al. 2000, Jenkins \& Hoffmann 2000, Radwan 2003). The results also show the trait specificity of stress-asymmetry associations which others have noted (e.g. Vøllestad et al. 1999, Woods et al. 1999, Hoffmann \& Woods 2003). One explanation for the apparent lack of interaction could be that under these conditions, i.e. inbreeding combined with thermal stress, pre-adult mortality is high (Fig. 3). As it has been proposed that more symmetrical individuals exhibit a higher fitness on average, the increased mortality observed under these conditions might have particularly affected the most asymmetrical individuals. This could certainly deflate the average levels of FA observed (Floate \& Fox 2000, Polak et al. 2002, 2004).

In conclusion, our findings suggest that increased levels of FA may indeed signal the presence of either genetic or thermal stress. An absence of inflated levels of FA, however, cannot be interpreted as the absence of stress. As such, we feel that FA is not a reliable biomonitor for conservation issues.

\subsection{Conclusions}

Habitat destruction and fragmentation, and climate change are among the most disquieting human activities that endanger global biodiversity. Apart from affecting many ecological parameters (Karlsson \& Van Dyck 2005, Pertoldi \& Bach 2007, Pearson et al. 2009), habitat fragmentation causes genetic drift and inbreeding, resulting in loss of genetic variation and a decrease in fitness (Bijlsma et al. 2000, Keller \& Waller 2002, Frankham 2005). The importance of the present study is that it shows that inbred populations are much more susceptible to thermal stress than non-inbred populations. The data presented in Fig. 3 clearly illustrate that thermal tolerance deflates significantly as a result of inbreeding. Whereas adaptive plasticity is crucial for a population to cope with the imposed stress in the short term, our results show that such responses can be significantly hampered by inbreeding. This idea is supported by recent data on the effect of inbreeding on the predator-induced adaptive responses in a freshwater snail (Auld \& Relyea 2010). As such, inbreeding enhances the harmful effects of stress on populations. Additionally, the loss of genetic variation accompanying habitat fragmentation can severely limit the adaptive potential of populations (Frankham 2005, Bell \& Gonzalez 2009, Bakker et al. 2010), thereby impairing evolutionary adaptation as a mechanism to cope with the effects of climate change (Willi et al. 2006, Kawecki 2008, Willi \& Hoffmann 2009).

In short, our results imply that the ongoing fragmentation of natural habitats will significantly enhance the adverse effects of climate change. Therefore, models developed to predict the consequences of climate change should preferably also consider the synergistic effects of habitat fragmentation. 
Acknowledgements. We are grateful to A. Boerema for technical assistance. We thank W. van Delden for helpful comments on the manuscript. This research was supported by the Research Council for Earth and Life Sciences (grant ALW 805.33.362-P), which is subsidized by the Netherlands Organization of Scientific Research (NWO). R.B. thanks the ESF Research Networking Programmes ConGen and ThermAdapt for financial support to attend the Białowieża workshop.

\section{LITERATURE CITED}

Armbruster P, Reed DH (2005) Inbreeding depression in benign and stressful environments. Heredity 95:235-242

Auld JR, Relyea RA (2010) Inbreeding depression in adaptive plasticity under predation risk in a freshwater snail. Biol Lett 6:222-4

Bakker J, Van Rijswijk MEC, Weissing FJ, Bijlsma R (2010) Consequences of fragmentation for the ability to adapt to novel environments in experimental Drosophila metapopulations. Conserv Genet 11:435-448

Bell G, Gonzalez A (2009) Evolutionary rescue can prevent extinction following environmental change. Ecol Lett 12: 942-948

Bijlsma R, Bundgaard J, Van Putten WF (1999) Environmental dependence of inbreeding depression and purging in Drosophila melanogaster. J Evol Biol 12:1125-1137

Bijlsma R, Bundgaard J, Boerema AC (2000) Does inbreeding affect the extinction risk of small populations? Predictions from Drosophila. J Evol Biol 13:502-514

Bijlsma R, Westerhof MDD, Roekx LP, Pen I (2010) Dynamics of genetic rescue in inbred Drosophila melanogaster populations. Conserv Genet 11:449-462

Brook BW, Tonkyn DW, O'Grady JJ, Frankham R (2002) Contribution of inbreeding to extinction risk in threatened species. Cons Ecol 6:16

Bubliy OA, Loeschke V, Imasheva AG (2000) Effect of stressful and nonstressful growth temperatures on variation of sternopleural bristle number in Drosophila melanogaster. Evolution 54:1444-1449

Canale CI, Henry PY (2010) Adaptive phenotypic plasticity and resilience of vertebrates to increasing climatic unpredictability. Clim Res 43:135-147

Chown SL, Gaston KJ (2008) Macrophysiology for a changing world. Proc R Soc Lond B 275:1469-1478

Chown SL, Hoffmann AA, Kristensen TN, Angiletta MJ Jr, Stenseth NC, Pertoldi C (2010) Adapting to climate change: a perspective from evolutionary physiology. Clim Res 43:3-15

Clarke GM (1995) Relationships between developmental stability and fitness: application for conservation biology. Conserv Biol 9:18-24

Dahlgaard J, Hoffmann AA (2000) Stress resistance and environmental dependency of inbreeding depression in Drosophila melanogaster. Conserv Biol 14:1187-1192

David JR, Allemand R, Van Herrewege J, Cohet Y (1983) Ecophysiology: abiotic factors. In: Ashburner M, Carson HL, Thompson JN (eds) The genetics and biology of Drosophila, Vol. 3. Academic Press, London, p 105-170

de Jong MA, Kesbeke FMNH, Brakefield PM, Zwaan BJ (2010) Geographic variation in thermal plasticity of life history and wing pattern in Bicyclus anynana. Clim Res 43:91-102

Deutsch CA, Tewksbury JJ, Huey RB, Sheldon KS, Ghalambor CK, Haak DC, Martin PR (2008) Impacts of climate warming on terrestrial ectotherms across latitude. Proc Natl Acad Sci USA 105:6668-6672
Falconer DS, Mackay TFC (1996) Introduction to quantitative genetics, 4th edn. Longmans Green, Harlow

Fernández J, Rodríguez-Ramilo ST, Pérez-Figueroa A, LópezFanjul C, Caballero A (2003) Lack of nonadditive genetic effects on early fecundity in Drosophila melanogaster. Evolution 57:558-565

Floate KD, Fox AS (2000) Flies under stress: a test of fluctuating asymmetry as a biomonitor of environmental quality. Ecol Appl 10:1541-1550

Fowler K, Whitlock MC (1999) The distribution of phenotypic variance with inbreeding. Evolution 53:1143-1156

Fowler K, Whitlock MC (2002) Environmental stress, inbreeding and the nature of phenotypic and genetic variance in Drosophila melanogaster. Proc R Soc Lond B 269:677-683

Frankham R (2005) Genetics and extinction. Biol Conserv 126: $131-140$

- Gaston KJ (2005) Biodiversity and extinction: species and people. Prog Phys Geogr 29:239-247

Gibert P, Capy P, Imasheva AG, Moreteau B, Morin JP, Petavy G, David JR (2004) Comparative analysis of morphological traits among Drosophila melanogaster and D. simulans: genetic variability, clines and phenotypic plasticity. Genetica 120:165-179

Gilligan DM, Woodworth LM, Montgomery ME, Nurthen RK, Briscoe DA, Frankham R (2000) Can fluctuating asymmetry be used to detect inbreeding and loss of genetic diversity in endangered populations? Anim Conserv 3:97-104

Gilpin ME, Soulé ME (1986) Minimum viable populations: processes of species extinction. In: Soulé ME (ed) Conservation biology: the science of scarcity and diversity. Sinauer, Sunderland, MA, p 19-34

- Hendry AP, Farrugia TJ, Kinnison MT (2008) Human influences in rates of phenotypic change in wild animal populations. Mol Ecol 17:20-29

Henle K, Lindenmayer DB, Margules CR, Saunders DA, Wissel C (2004) Species survival in fragmented landscapes: Where are we now? Biodivers Conserv 13:1-8

Hoffmann AA, Woods RE (2003) Associating environmental stress with developmental stability: problems and patterns. In: Polak M (ed) Developmental instability: causes and consequences. Oxford University Press, New York, p 387-401

> Hoffmann AA, Sørensen JG, Loeschke V (2003) Adaptation of Drosophila to temperature extremes: bringing together quantitative and molecular approaches. J Therm Biol 28: $175-216$

> Hoffmeister TS, Vet LEM, Biere A, Holsinger K, Filser J (2005) Ecological and evolutionary consequences of biological invasion and habitat fragmentation. Ecosystems 8:657-667

Hosken DJ, Blanckenhorn WU, Ward PI (2000) Developmental stability in yellow dung flies (Scathophaga stercoraria): fluctuating asymmetry, heterozygosity and environmental stress. J Evol Biol 13:919-926

Imasheva AG, Loeschke V, Zhivotovsky LA, Lazebny OE (1997) Effects of extreme temperatures on phenotypic variation and developmental stability in Drosophila melanogaster and Drosophila buzzatii. Biol J Linn Soc 61: $117-126$

Imasheva AG, Loeschke V, Zhivotovsky LA, Lazebny OE (1998) Stress temperatures and quantitative variation in Drosophila melanogaster. Heredity 81:246-253

IPCC (2007) Climate change 2007: impacts, adaptation and vulnerability. Contribution of Working Group II to the Fourth Assessment Report of the Intergovernmental Panel on Climate Change. Cambridge University Press, Cambridge

Jenkins NL, Hoffmann AA (2000) Variation in morphological 
traits and trait asymmetry in field Drosophila serrata from marginal populations. J Evol Biol 13:113-130

Joubert D (2007) Fluctuating asymmetry as a monitor for genetic and environmental stress in Drosophila: fundamental and applied aspects. PhD dissertation, University of Groningen

> Karan D, Morin JP, Moreteau B, David JR (1998) Body size and developmental temperature in Drosophila melanogaster: analysis of body weight reaction norm. J Therm Biol 23:301-309

Karlsson B, Van Dyck H (2005) Does habitat fragmentation affect temperature-related life-history traits? A laboratory test with a woodland butterfly. Proc R Soc Lond B 272: $1257-1263$

Kawecki TJ (2008) Adaptation to marginal habitats. Annu Rev Ecol Evol Syst 39:321-342

Keller LF, Waller DM (2002) Inbreeding effects in wild populations. Trends Ecol Evol 17:230-241

Kellermann V, van Heerwarden B, Sgrò CM, Hoffmann AA (2009) Fundamental evolutionary limits in ecological traits drive Drosophila species distributions. Science 325: 1244-1246

Kjærsgaard A, Faurby S, Krag K, Loeschke V, Pertoldi C (2010) Temperature-maternal age interactions on wing traits in outbred Drosophila mercatorum. Clim Res 43: 49-56

Kristensen TN, Dahlgaard J, Loeschke V (2003) Effects of inbreeding and environmental stress on fitness - using Drosophila buzzatii as a model organism. Conserv Genet 4:453-465

Kristensen TN, Barker JSF, Pedersen KS, Loeschke V (2008a) Extreme temperatures increase the deleterious consequences of inbreeding under laboratory and semi-natural conditions. Proc R Soc Lond B 275:2055-2061

Kristensen TN, Loeschke V, Hoffmann AA (2008b) Linking inbreeding effects in captive populations with fitness in the wild: release of replicated Drosophila melanogaster lines under different temperatures. Conserv Biol 22: 189-199

Kristensen TN, Hoffmann AA, Overgaard J, Sørensen JG, Hallas R, Loeschke V (2008c) Costs and benefits of cold acclimation in field-released Drosophila. Proc Natl Acad Sci USA 105:216-221

Lens L, Van Dongen S, Matthyssen E (2002) Fluctuating asymmetry as an early warning system in the critically endangered Taita thrush. Conserv Biol 16:479-487

Lerner IM (1954) Genetic homeostasis. John Wiley and Sons, New York

Leung B, Knopper L, Mineau P (2003) A critical assessment of the utility of fluctuating asymmetry as a biomarker of anthropogenic stress. In: Polak M (ed) Developmental instability: causes and consequences. Oxford University Press, New York, p 415-426

Liao W, Reed DH (2009) Inbreeding-environment interactions increase extinction risks. Anim Conserv 12:54-61

Lynch M, Walsh JB (1998) Genetics and analysis of quantitative traits. Sinauer, Sunderland, MA

- Mawdsley JR, O'Malley R, Ojima DS (2009) A review of climate-change adaptation strategies for wildlife management and biodiversity conservation. Conserv Biol 23: $1080-1089$

> Moreteau B, Gibert P, Delpuech JM, Petavy G, David JR (2003) Phenotypic plasticity of sternopleural bristle number in temperate and tropical populations of Drosophila melanogaster. Genet Res 81:25-32

> Mousseau TA, Roff DA (1987) Natural selection and the heritability of fitness components. Heredity 59:181-197
Nunney L (1996) The response to selection for fast larval development in Drosophila melanogaster and its effect on adult weight: an example of a fitness trade-off. Evolution 50:1193-1204

Palmer AR (1994) Fluctuating asymmetry analyses: a primer. In: Markow TA (ed) Developmental instability: its origins and evolutionary implications. Kluwer Academic Publishers, Dordrecht, p 335-364

> Parsons PA (1992) Fluctuating asymmetry: a biological monitor of environmental and genomic stress. Heredity 68:361-364

Pearson GA, Lago-Leston A, Mota C (2009) Frayed at the edges: selective pressure and adaptive response to abiotic stressors are mismatched in low diversity edge populations. J Ecol 97:450-462

Pedersen KS, Kristensen TN, Loeschke V (2005) Effects of inbreeding and rate of inbreeding in Drosophila melanogaster-Hsp70 expression and fitness. J Evol Biol 18: 756-762

Pertoldi C, Bach LA (2007) Evolutionary aspects of climateinduced changes and the need for multidisciplinarity. J Therm Biol 32:118-124

> Pertoldi C, Kristensen TN, Andersen DH, Loeschke V (2006) Developmental instability as an estimator of genetic stress. Heredity 96:122-127

> Polak M, Opoka R, Cartwright IL (2002) Response of fluctuating asymmetry to arsenic toxicity: support for the developmental selection hypothesis. Environ Pollut 118:19-28

Polak M, Kroeger DE, Cartwright IL, Ponce deLeon C (2004) Genotype-specific responses of the effects of lead and temperature stress in Drosophila melanogaster. Environ Pollut 127:145-155

Radwan J (2003) Inbreeding not stress increases fluctuating asymmetry in the bulb mite. Evol Ecol Res 5:287-295

$>$ Reed DH, Briscoe DA, Frankham R (2002) Inbreeding and extinction: the effect of environmental stress and lineage. Conserv Genet 3:301-307

- Rice WR (1989) Analyzing tables of statistical tests. Evolution 43:223-225

> Root TL, Schneider SH (2006) Conservation and climate: the challenges ahead. Conserv Biol 20:706-708

> Saccheri I, Kuussaari M, Kankare M, Vikman P, Fortelius W, Hanski I (1998) Inbreeding and extinction in a butterfly metapopulation. Nature 392:491-494

Thomas CD, Cameron A, Green RE, Bakkenes M and others (2004) Extinction risk from climate change. Nature 427: $145-148$

Trotta V, Pertoldi C, Rudoy A, Manenti T, Cavicchi S, Guerra D (2010) Thermal plasticity of wing size and shape in Drosophila melanogaster, D. simulans and their hybrids. Clim Res 43:71-79

Van Buskirk J, Willi Y (2006) The change in quantitative genetic variation with inbreeding. Evolution 60:2428-2434

Van Delden W, Beardmore JA (1968) Effects of small increments of genetic variability in inbred populations of Drosophila melanogaster. Mutat Res 6:117-127

Vermeulen CJ, Bijlsma R (2004a) Changes in mortality patterns and temperature dependence of lifespan in Drosophila melanogaster caused by inbreeding. Heredity 92: 275-281

Vermeulen CJ, Bijlsma R (2004b) Characterization of conditionally expressed mutants affecting age-specific survival in inbred lines of Drosophila melanogaster: lethal conditions and temperature-sensitive periods. Genetics 167 : 1241-1248

Vøllestad LA, Hindar K, Møller AP (1999) A meta-analysis of fluctuating asymmetry in relation to heterozygosity. Heredity 83:206-218 
Waddington $\mathrm{CH}$ (1957) The strategy of the genes. George Allen \& Unwin, London

Walther GR, Post E, Convey P, Menzel A and others (2002) Ecological responses to recent climate change. Nature 416:389-395

Whitlock MC, Fowler K (1999) The changes in genetic and environmental variance with inbreeding in Drosophila melanogaster. Genetics 152:345-353

> Willi Y, Hoffmann AA (2009) Demographic factors and genetic variation influence persistence under environmental change. J Evol Biol 22:124-133

Willi Y, Van Buskirk J, Hoffmann AA (2006) Limits to the adaptive potential of small populations. Annu Rev Ecol Evol Syst 37:433-458

Submitted: April 6, 2010; Accepted: May 19, 2010
Woods RE, Sgrò CM, Hercus MJ, Hoffmann AA (1999) The association between fluctuating asymmetry, trait variability, trait heritability, and stress: a multiply replicated experiment on combined stresses in Drosophila melanogaster. Evolution 53:493-505

Wright S (1977) Evolution and the genetics of populations, Vol 3. Experimental results and evolutionary deductions. University of Chicago Press, Chicago, IL

Zar J (1996) Biostatistical analysis, 3rd edn. Prentice Hall, Upper Saddle River, NJ

Zwaan B, Bijlsma R, Hoekstra RF (1995) Artificial selection for developmental time in Drosophila melanogaster in relation to the evolution of aging: direct and correlated responses. Evolution 49:635-648

Proofs received from author(s): June 28, 2010 